\title{
Effectiveness of the Benign and Malignant Diagnosis of Mediastinal and Hilar Lymph Nodes by Endobronchial Ultrasound Elastography
}

\author{
Haidong Huang $1^{*}$, Zhiang Huang ${ }^{2^{*}}$, Qin Wang ${ }^{1^{*}}$, Xinan Wang $3^{3^{*}}$, Yuchao Dong1, Wei Zhang ${ }^{1}$, Paul \\ Zarogoulidis $^{4 \bowtie}$, Yan-Gao Man ${ }^{5}$, Wolfgang Hohenforst Schmidt ${ }^{6}$, Chong Bai ${ }^{1 凶}$ \\ 1. Department of Respiratory \& Critical Care Medicine, Changhai Hospital, the Second Military Medical University, China \\ 2. Department of Respiratory, The first affiliated hospital of henan university, China. \\ 3. Department of Respiratory Medicine, Binzhou People 's Hospital, China \\ 4. Pulmonary Oncology Unit, “G. Papanikolaou” General Hospital, Aristotle University of Thessaloniki, Thessaloniki, Greece \\ 5. Research Laboratory and International Collaboration, Bon Secours Cancer Institute, VA, USA \\ 6. Sana Clinic Group Franken, Department of Cardiology / Pulmonology / Intensive Care / Nephrology, "Hof" Clinics, University of Erlangen, Hof, Germany \\ * Haidong Huang, Zhiang Huang, Qin Wang, Xinan Wang, equally contributed to this work. \\ $\triangle$ Corresponding authors: Paul Zarogoulidis, M.D, Ph. D, Pulmonary Department-Oncology Unit, "G. Papanikolaou” General Hospital, Aristotle University of \\ Thessaloniki, Thessaloniki, Greece. Fax: 00302310992424; Mobile: 00306977271974; E-mail: zarog@hotmail.com; Chong Bai, Department of Respiratory \& Critical Care \\ Medicine, Changhai Hospital, the Second Military Medical University, China. \\ (c) Ivyspring International Publisher. This is an open access article distributed under the terms of the Creative Commons Attribution (CC BY-NC) license \\ (https://creativecommons.org/licenses/by-nc/4.0/). See http://ivyspring.com/terms for full terms and conditions.
}

Received: 2017.02.26; Accepted: 2017.05.03; Published: 2017.07.02

\begin{abstract}
Background and Objectives: Endobronchial ultrasound elastography is a new technique for describing the stiffness of tissue during endobronchial ultrasound-guided transbronchial needle aspiration (EBUS-TBNA). The aims of this study were to investigate the diagnostic value of Endobronchial ultrasound (EBUS) elastography for distinguishing the difference between benign and malignant lymph nodes among mediastinal and hilar lymph nodes.

Materials and Methods: From June 2015 to August 2015, 47 patients confirmed of mediastinal and hilar lymph node enlargement through examination of Computed tomography (CT) were enrolled, and a total of 78 lymph nodes were evaluated by endobronchial ultrasound-guided transbronchial needle aspiration (EBUS-TBNA). EBUS-guided elastography of lymph nodes was performed prior to EBUS-TBNA. A convex probe EBUS was used with a new EBUS processor to assess elastographic patterns that were classified based on color distribution as follows: Type 1, predominantly non-blue (green, yellow and red); Type 2, part blue, part non-blue (green, yellow and red); Type 3, predominantly blue. Pathological determination of malignant or benign lymph nodes was used as the gold standard for this study. The elastographic patterns were compared with the final pathologic results from EBUS-TBNA.

Results: On pathological evaluation of the lymph nodes, 45 were benign and 33 were malignant. The lymph nodes that were classified as Type 1 on endobronchial ultrasound elastography were benign in $26 / 27$ (96.3\%) and malignant in 1/27 (3.7\%); for Type 2 lymph nodes, $15 / 20$ (75.0\%) were benign and 5/20 (25.0\%) were malignant; Type 3 lymph nodes were benign in 4/31 (12.9\%) and malignant in 27/31 (87.1\%). In classifying Type 1 as 'benign' and Type 3 as 'malignant,' the sensitivity, specificity, positive predictive value, negative predictive value and diagnostic accuracy rates were $96.43 \%, 86.67 \%, 87.10 \%, 96.30 \%, 91.38 \%$, respectively.

Conclusion: EBUS elastography of mediastinal and hilar lymph nodes is a noninvasive technique that can be performed reliably and may be helpful in the prediction of benign and malignant lymph nodes among mediastinal and hilar lymph node during EBUS-TBNA.
\end{abstract}

Key words: Bronchoscope, Ultrasound Elastography, Mediastinal and Hilar Lymph Nodes, Diagnosis

\section{INTRODUCTION}

Recently, elastography, a new ultrasonographyassociated technology that measures tissue compressibility, was introduced in the market. In principle, pathophysiological processes, such as malignancy, make tissues less deformable or stiff. 
Compression of surrounding structures produces a deformity or strain effect that is inversely related to the hardness of the pathologic tissue; harder tissues are less deformable than softer tissues. So ultrasound elastography can be used to identify benign and malignant tissue through measuring tissue compressibility. There have been several reports suggesting that endoscopic ultrasound (EUS) elastography had a high sensitivity and specificity for detecting malignant involvement of LNs, breast and thyroid disease ${ }^{[1-6]}$. However, the role of ultrasound elastography in the diagnosis of mediastinal and hilar lymph nodes is still unknown. In our present study we aimed to investigate the diagnostic value of Endobronchial ultrasound (EBUS) elastography for distinguishing benign and malignant lymph nodes in the mediastinum.

\section{MATERIALS AND METHODS}

\section{Patients and Methods}

\section{Patients}

This was a retrospective study with patients who underwent elastography with EBUS-TBNA at our institution between 1 June 2015 and 31 August 2015 with an Olympus ${ }^{\circledR}$ EBUS system. At least one enlarged mediastinal or hilar lymphadenopathy (short axis greater than $1 \mathrm{~cm}$ ) was observed in all patients on CT chest images. EBUS-TBNA was performed for clinical reasons independent of the purposes of the study. Here, 47 patients confirmed of mediastinal and hilar lymph node enlargement through examination of Computed tomography (CT) were enrolled, and a total of 78 lymph nodes were evaluated by endobronchial ultrasound-guided transbronchial needle aspiration (EBUS-TBNA) 22G EBUS needle. EBUS-guided elastography of lymph nodes was performed prior to EBUS-TBNA. We had collected 47 patients with mediastinal or hilar lymph node(s) enlargement(s), of which 29 males and 18 females, aged 25-77, with an average age of $60.19 \pm$ 11.03. All patients gave written informed consent before the procedure. Institutional review board approval was granted for this retrospective review.

\section{EBUS-TBNA procedure}

The patients should complete 6-8 hours of fasting, routine blood test, thin-slice CT scans of lungs, and electrocardiogram before the examination, patients were placed under moderate to deep sedation, which was preferred for the patients after evaluation of the anesthesiologist. Patient was given oropharyngeal anesthesia by oral inhalation of $2 \mathrm{ml}$ of $2 \%$ lidocaine injection. Then, the patient was administered inducing dose of dexmedetomidine (1 $\mu \mathrm{g} / \mathrm{kg}$ ) via micro pump of superficial vein in 10-15 minutes. After that, a dose of $0.5-0.7 \mu \mathrm{g} \cdot \mathrm{kg}^{-1} \mathrm{~h}^{-1}$ was given for maintenance. After falling asleep, the patients were administrated midazolam (0.03-0.05 mg.kg-1) and fentanyl (25-50 $\mu \mathrm{g})$ through slow intravenous push. If necessary, the dosage of midazolam and fentanyl could be increased as appropriate. We inserted nasopharyngeal tube to keep the upper respiratory tract unobstructed. For the patients with contraindications, such as uncontrolled hypertension and arrhythmia, local anesthesia of oropharyngeal inhaled aerosolized lidocaine should be only applied alternatively. The convex probe EBUS (BF-UC260F, Olympus, Japan) was inserted through the nose route, with intermittent instillation of $2 \mathrm{ml}$ aliquot doses of 2\% lidocaine. Elastography was performed on all LNs that were candidates for EBUS-TBNA. The elasticity of tissue within the scanned area was reconstructed by comparing it with the surrounding tissue, and translated this into a color signal that overlaid the B-mode image. The colors associated with hard, intermediate and soft tissues were blue, green and yellow/red, respectively. The complete spectrum from blue to red encoding was applied to each elastographic record and indicated the calibration of relative elasticity of the scanned area. Elastographic and B-mode images were simultaneously displayed on the monitor side by side. After elastography evaluation, EBUS-TBNA (NA-201SX-4022, Olympus ${ }^{\circledR}$, Japan) with negative pressure was done for 20-30 times, under real-time EBUS guidance. Histology and cytology specimens were collected accordingly. Rapid on-site cytology evaluation (ROSE) was performed for every case. The final diagnosis was confirmed from independent pathological examination of EBUS-TBNA specimens by pathologists who did not know the results of EBUS elastography.

\section{Statistical Analysis}

Statistical analyses were performed using spss18.0 statistical analysis software. Statistical significance between groups was determined by $\chi^{2}$ test. Differences were considered to be significant when $\mathrm{p}<0.05$.

\section{RESULTS}

\section{Baseline characteristics of the lymph nodes}

The baseline characteristics of all the LNs evaluated in this study were summarized in Table 1 and Table 2. There were 47 patients who underwent elastography with EBUS-TBNA of 78 hilar and mediastinal LNs. 
Table 1: Distribution of Lymph node number of patients with mediastinal or hilar lymph node(s) enlargement(s) in Changhai Hospital during June 2015 to August 2015

\begin{tabular}{lll}
\hline Number/patients & Patients & Size,short axis $(\mathrm{mm})$ \\
\hline 1 & 27 & $20.88 \pm 6.99$ \\
2 & 12 & $21.98 \pm 8.76$ \\
3 & 5 & $13.45 \pm 3.03$ \\
4 & 3 & $21.14 \pm 6.13$ \\
\hline
\end{tabular}

Table 2: Distribution of Lymph node station of patients with mediastinal or hilar lymph node(s) enlargement(s) in Changhai Hospital during June 2015 to August 2015

\begin{tabular}{lll}
\hline Lymph node station & Number & Size, short axis $(\mathrm{mm})$ \\
\hline $4 \mathrm{R}$ & 29 & $18.81 \pm 7.38$ \\
$4 \mathrm{~L}$ & 6 & $24.63 \pm 9.58$ \\
7 & 20 & $21.79 \pm 7.41$ \\
$11 \mathrm{R}$ & 9 & $21.56 \pm 7.76$ \\
$11 \mathrm{~L}$ & 14 & $15.97 \pm 5.26$ \\
\hline
\end{tabular}

\section{Representative lymph nodes on EBUS elastography}

Classification of LNs was performed. Elastographic patterns were described according to the dominant colors and their distribution within the target LN. This description formed the basis for the following classification of elastographic types: Type 1 [Figure 1A]: predominantly non-blue (green, yellow and red). Type 2 [Figure 1B]: part blue, part non-blue (green, yellow and red). Type 3 [Figure 1C]: predominantly blue. Only LNs with adequate lymphocytes or those with a definitive diagnosis were included for data analysis.

\section{Pathological examinations of the lymph node}

47 patients confirmed of mediastinal and hilar lymph node enlargement through examination of Computed tomography (CT) were enrolled, and a total of 78 lymph nodes were evaluated by endobronchial ultrasound-guided transbronchial needle aspiration (EBUS-TBNA). EBUS-guided elastography of lymph nodes was performed prior to EBUS-TBNA. On pathological evaluation of the lymph nodes, 45 were benign and 33 were malignant. Among benign lymph nodes, there are normal lymph tissue in 20 cases, and epithelioid granuloma lesions in 25 cases. Among malignant lymph nodes, there are small cell carcinoma in 7 cases, adenocarcinoma in 21 cases, squamous carcinoma in 2 cases, between the cytopathology of malignant tumor in 1 case, diffuse large B cell lymphoma in 1 case and carcinoid tumor in 1 case.

\section{EBUS elastography classification of lymph nodes}

Pathological determination of malignant or benign lymph nodes was used as the gold standard for this study. The elastographic patterns were compared with the final pathologic results from EBUS-TBNA [Table 3]. The lymph nodes that were classified as Type 1 on endobronchial ultrasound elastography were benign in $26 / 27(96.3 \%)$ and malignant in 1/27 (3.7\%); for Type 2 lymph nodes, $15 / 20(75.0 \%)$ were benign and $5 / 20(25.0 \%)$ were malignant; Type 3 lymph nodes were benign in 4/31 $(12.9 \%)$ and malignant in $27 / 31(87.1 \%)$. In classifying Type 1 as 'benign' and Type 3 as 'malignant,' the sensitivity, specificity, positive predictive value, negative predictive value and diagnostic accuracy rates were $96.43 \%, 86.67 \%, 87.10 \%, 96.30 \%, 91.38 \%$, respectively.

Table 3: EBUS elastography classification of lymph nodes

\begin{tabular}{lll}
\hline Elastography type & $\begin{array}{l}\text { Number of malignant } \\
\text { LNs/total number }(\%)\end{array}$ & $\begin{array}{l}\text { Number of benign } \\
\text { LNs/total number }(\%)\end{array}$ \\
\hline Type $1(\mathrm{n}=27)$ & $1 / 27(3.7 \%)$ & $26 / 27(96.3 \%)$ \\
Type 2 $(\mathrm{n}=20)$ & $5 / 20(25.0 \%)$ & $15 / 20(75.0 \%)$ \\
Type 3 $(\mathrm{n}=31)$ & $27 / 31(87.1 \%)$ & $4 / 31(12.9 \%)$ \\
\hline
\end{tabular}

\section{DISCUSSION}

Recently, Endobronchial ultrasound guided transbronchial needle aspiration (EBUS-TBNA) is a widely used minimally invasive procedure that has been shown to have a high sensitivity and diagnostic yield for not only detecting metastasis to hilar and mediastinal lymph nodes (LNs) but also in lung cancer staging and diagnosis ${ }^{[7,8]}$. Though, there have been several reports suggesting that endoscopic ultrasound (EUS) elastography had a high sensitivity and specificity for detecting malignant involvement of LNs, breast and thyroid disease. Subsequently, elastography has become available for use during EBUS[9]. As of yet, evidence is needed to assess whether elastography can be a valuable tool in the noninvasive discrimination between benign and malignant Mediastinal and Hilar LNs during EBUS-TBNA. This study found the utility of elastography for hilar and mediastinal LNs during EBUS-TBNA.

Based on our results, we propose a simple EBUS elastography classification that had been reported by Takehiro lzumo[10]. Type 1 (predominantly non-blue) indicates a benign pathology; Type 2 (part blue, part non-blue) is equivocal; and Type 3 (predominantly blue) indicates malignancy. The lymph nodes that were classified as Type 1 on endobronchial ultrasound elastography were normal lymph tissue in 16/27 (59.26\%), granulomatous lesions lymphoid tissue in 10/27 (37.04\%) and malignant in 1/27 (3.7\%), so Type 1 lymph nodes could be stored temporarily in 
observation if there is no obvious puncture indications because of high negative predictive value. For Type 2 lymph nodes, we found normal lymph

A

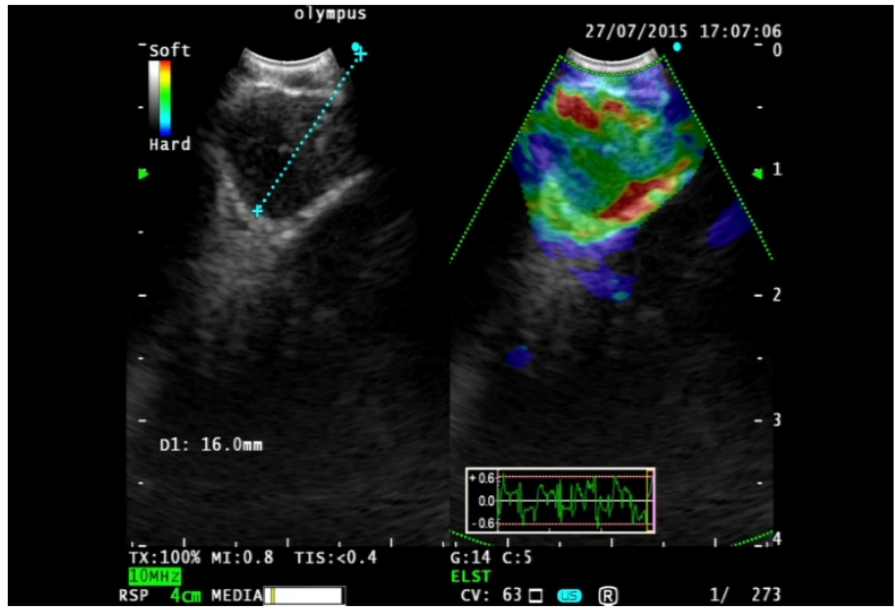

$\mathrm{B}$

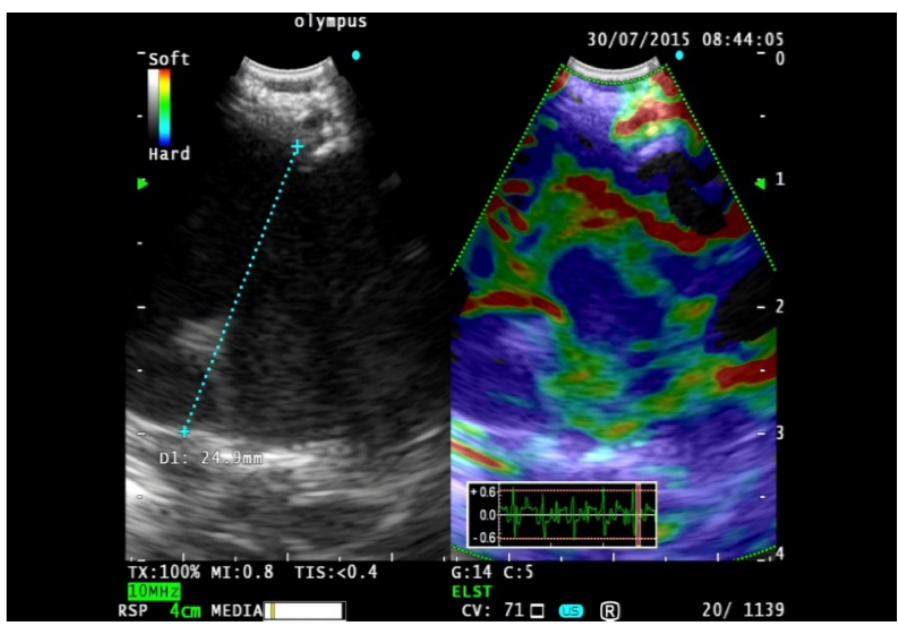

$\mathrm{C}$

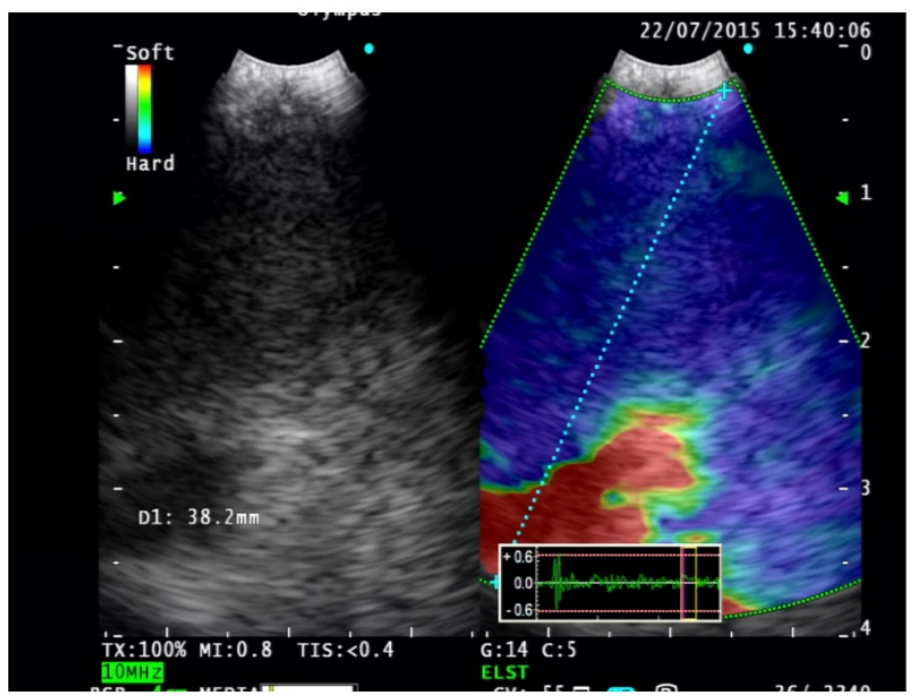

Figure 1: A: Type 1 predominantly non-blue (green, yellow and red). B: Type 2 part blue, part non-blue (green, yellow and red). C: Type 3 predominantly blue. tissue in $1 / 20(5 \%)$, granulomatous lesions lymphoid tissue in 14/20 (70\%) and malignant in 5/20 (25\%). Though this lesion type shows higher NPV than PPV, it has a relevant malignancy rate. We suggest that lymph nodes of this type 2 should be needled in any case. Type 3 lymph nodes have the highest positive predictive value $(87.1 \%)$ and mostly are malignant disease based on pathological results. For Type 3 elastography LNs, the high positive predictive value could help in promptly deciding a target area to sample during EBUS-TBNA. But this method in estimating area percentage, easily influenced by subjective factors, therefore, will produce certain error. In 2015, Takahiro Nakajima etc. reported a new EBUS elastography classification method that can be more accurate in classification of lymph nodes through image J1.45 software calculating the pixel area and then acquire the radio of hard lymph node (blue) area to avoid the classification error due to the subjective factors effect in sonographic view of each object lymph node ${ }^{[11]}$. But, there still is a certain error of the judgment of the whole lymph node hardness, because the software calculation area is only for one sonographic view of lymph node, the elastography classification method need to be further improved.

As one result of this study we found false positive LNs in the Type 3 group classified histologically as sarcoidosis [Figure 2], due to fibrous tissue hyperplasia in lymphoid tissue leading to increased hardness. For false negative patients in type 1 lymph nodes, the final pathologic result was adenocarcinoma [Figure 3], because the lymph node invasion by tumor cells caused partial lymphoid tissue liquefaction. Due to the above-mentioned reasons, operators should combine clinical examination with patients medical history in order to avoid misdiagnosis. There are two groups of patients; one is tuberculosis patients, with caseous necrosis and more calcifications which increase organization hardness. The second is chronic lymphadenitis patients: Here the tissue hardens by increased fibrous tissue hyperplasia. In these 2 groups the operator should pay high attention during EBUS elastography examination of superficial lymph nodes. 


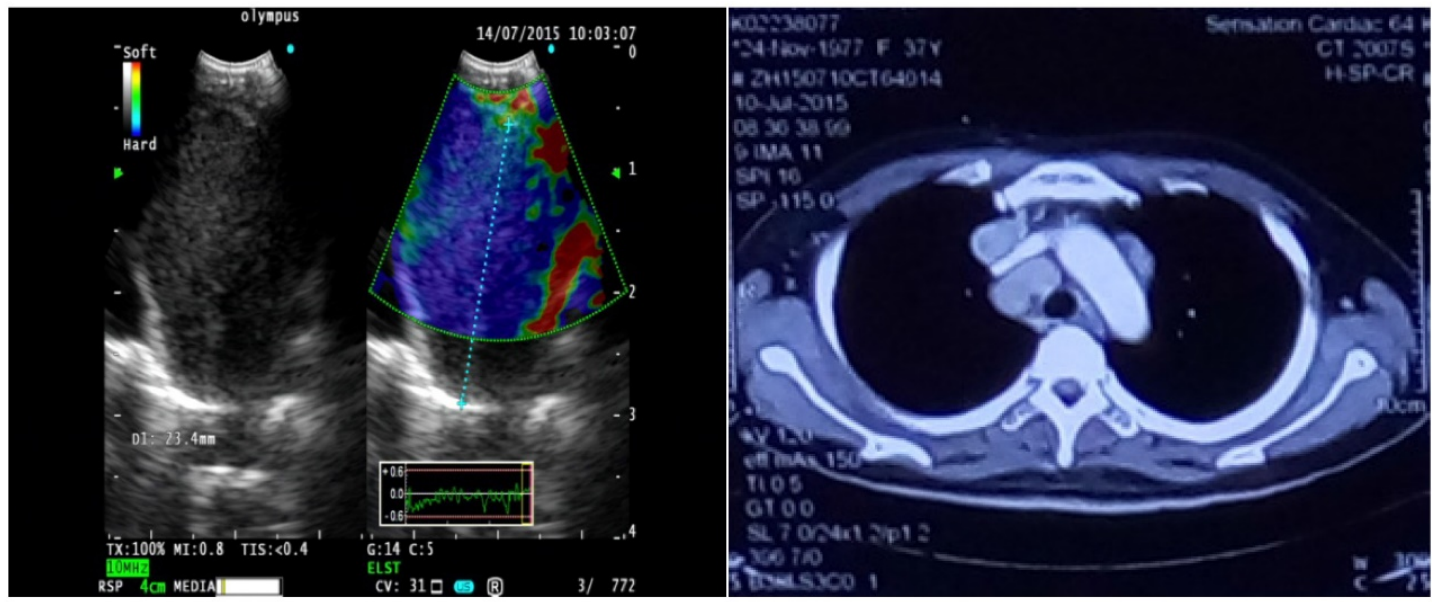

Figure 2: A case of 4R with Type 1 lymph node on EBUS elastography, pathological results show scattered granulomas, did not clear tumor lesions, considering sarcoidosis.

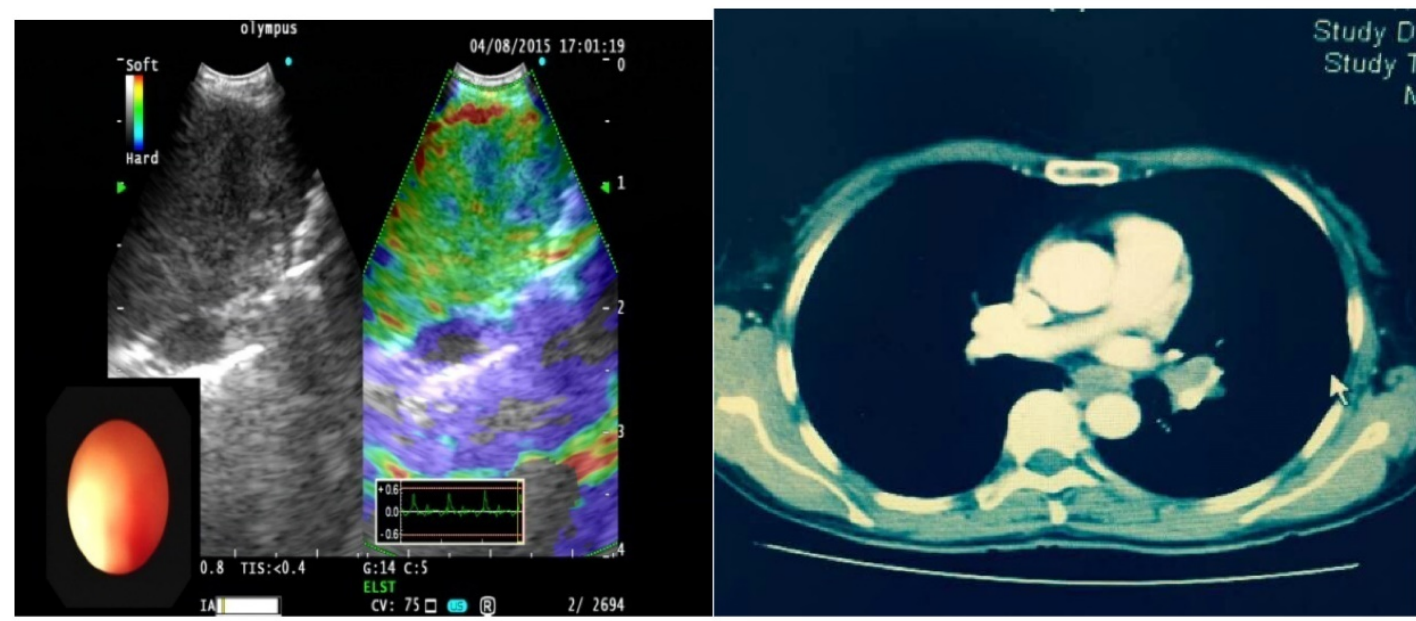

Figure 3: A case of $11 \mathrm{~L}$ with Type 1 lymph node on EBUS elastography, pathological results show poorly differentiated adenocarcinoma.

\section{CONCLUSION}

In 2010, Taiki Fujiwara had studied the benign and malignant mediastinal lymph nodes in six aspects, such as size, shape, boundary, echo of lymph nodes, hilus and coagulation necrosis ${ }^{[12]}$. In 2012, Takahiro Nakajima etc. had studied the relationship of blood supply between benign and malignant lymph nodes ${ }^{[13]}$. The final results indicated that the sensitivity is not ideal. By ultrasonic bronchoscopy elastography in the lung and mediastinal lymph node, the application of the study found that the sensitivity and specificity between benign and malignant diagnosis when compared with the previous two methods is improved greatly and provides us with a new diagnostic tool.

In order to provide better guidance for clinical doctors, EBUS elastography classification of lymph nodes still needs further exploration, development and improvement as it lacks of unified standards. So on the basis of EBUS elastography classification of lymph nodes, we should further combine patients history and clinical examination in order to interpret correctly the pathological results. In addition with the increasing development of technology, we believe that EBUS elastography will be more and more used in the everyday practice and will play a basic role in the diagnostic approach of benign and malignant mediastinal lymph nodes ${ }^{[14]}$.

\section{Acknowledgements}

This study was supported by Project of New Clinical Technology Development of Changhai Hospital (Grant number: NT201506), Project of Wujieping Medical Foundation (Grant number: 320.6750.11112), and Project of Shanghai Health and Family Planning Commission Foundation (Grant number: 20124166). 


\section{Competing Interests}

There are no conflicts of interest.

\section{References}

1. Moon WK, Chang RF, Chen CJ, et al. Solid breast masses: classification with computer-aided analysis of continuous US images obtained with probe compression. Radiology 2005; 236: 458-64.

2. Lyshchik A, Higashi T, Asato R, et al. Thyroid gland tumor diagnosis at US elastography. Radiology 2005; 237: 202-11.

3. Foucher J, Chanteloup E, Vergniol J, et al. Diagnosis of cirrhosis by transient elastography (FibroScan): a prospective study. Gut 2006; 55: 403-8.

4. Garra BS, Cespedes EI, Ophir J, et al. Elastography of breast lesions: initial clinical results. Radiology 1997; 202: 79-86.

5. Giovannini M, Hookey LC, Bories E, et al. Endoscopic ultrasound elastography: the first step towards virtual biopsy Preliminary results in 49 patients. Endoscopy 2006; 38: 344-8.

6. Alam F, Naito K, Horiguchi J, et al. Accuracy of sonographic elastography in the differential diagnosis of enlarged cervical lymph nodes: comparison with conventional B-mode sonography. AJR Am J Roentgenol 2008; 191: 604-10.

7. Rivera MP, Mehta AC, Wahidi MM. Establishing the diagnosis of lung cancer: diagnosis and management of lung cancer, 3rd ed: American College of Chest Physicians evidence-based clinical practice guidelines. Chest 2013; 143: e142S-65S.

8. Yasufuku K, Nakajima T, Waddell T, et al. Endobronchial ultrasound-guided transbronchial needle aspiration for differentiating N0 versus N1 lung cancer. Ann Thorac Surg 2013; 96: 1756-60.

9. Trosini-Desert V, Jeny F, Taillade L, et al. Bronchial endoscopic ultrasound elastography: preliminary feasibility data. Eur Respir J 2013; 41: 477-9.

10. Takehiro Izumo, Shinji Sasada, Christine Chavez, et al. Endobronchial Ultrasound Elastography in the Diagnosis of Mediastinal and Hilar Lymph Nodes. Ipn J Clin Oncol 2014; 44 (10): 956-962.

11. Takahiro Nakajima, et al. The Role of Ultrasonographic Imaging Analysis during EBUS-TBNA. WABIP Newsletter 2015; 3(2).

12. Taiki Fujiwara, Kazuhiro Yasufuku, Takahiro Nakajima, et al. The Utility of Sonographic Features During Endobronchial Ultrasound-Guided Transbronchial Needle Aspiration for Lymphnode Staging in Patients With Lung Cancer. Chest 2010; 138 (3): 641-647.

13. Takahiro Nakajima, Takashi Anayama, Masato Shingyoji, et al. Vascular Image Patterns of Lymph Nodes for the Prediction of Metastatic Disease During EBUS-TBNA for Mediastinal Staging of Lung Cancer. J Thorac Oncol 2012; 7 (6): 1009-1014.

14. Dietrich CF, Jenssen C, Herth FJ. Endobronchial ultrasound elastography. Endosc Ultrasound 2016; 5(4):233-238. 